This item was submitted to Loughborough's Research Repository by the author.

Items in Figshare are protected by copyright, with all rights reserved, unless otherwise indicated.

\title{
A high performance biometric signal and image processing method to reveal blood perfusion towards 3D oxygen saturation mapping
}

PLEASE CITE THE PUBLISHED VERSION

http://dx.doi.org/10.1117/12.2044318

\section{PUBLISHER}

(C) Society of Photo-Optical Instrumentation Engineers

\section{VERSION}

VoR (Version of Record)

\section{PUBLISHER STATEMENT}

This work is made available according to the conditions of the Creative Commons Attribution-NonCommercialNoDerivatives 4.0 International (CC BY-NC-ND 4.0) licence. Full details of this licence are available at: https://creativecommons.org/licenses/by-nc-nd/4.0/

\section{LICENCE}

CC BY-NC-ND 4.0

\section{REPOSITORY RECORD}

Imms, Ryan, Sijung Hu, Vicente Azorin-Peris, Michael Trico, and Ron Summers. 2019. "A High Performance Biometric Signal and Image Processing Method to Reveal Blood Perfusion Towards 3D Oxygen Saturation Mapping". figshare. https://hdl.handle.net/2134/21293. 


\title{
A high performance biometric signal and image processing method to reveal blood perfusion towards 3D oxygen saturation mapping
}

\author{
Ryan Imms ${ }^{a}$, Sijung $\mathrm{Hu}^{\mathrm{a} *}$, Vicente Azorin-Peris ${ }^{a}$, Michaël Trico ${ }^{b}$, Ron Summers ${ }^{a}$ \\ ${ }^{a}$ School of Electronic, Electrical and Systems Engineering, Loughborough University, Ashby Road, \\ Loughborough, Leicestershire, LE11 3TU, UK; \\ ${ }^{\mathrm{b}}$ Polytech Paris Sud Engineering School, Paris Sud University - bat 620, 91440 ORSAY, FRANCE
}

\begin{abstract}
Non-contact imaging photoplethysmography (PPG) is a recent development in the field of physiological data acquisition, currently undergoing a large amount of research to characterize and define the range of its capabilities. Contact-based PPG techniques have been broadly used in clinical scenarios for a number of years to obtain direct information about the degree of oxygen saturation for patients. With the advent of imaging techniques, there is strong potential to enable access to additional information such as multi-dimensional blood perfusion and saturation mapping. The further development of effective opto-physiological monitoring techniques is dependent upon novel modelling techniques coupled with improved sensor design and effective signal processing methodologies. The biometric signal and imaging processing platform (bSIPP) provides a comprehensive set of features for extraction and analysis of recorded iPPG data, enabling direct comparison with other biomedical diagnostic tools such as ECG and EEG. Additionally, utilizing information about the nature of tissue structure has enabled the generation of an engineering model describing the behaviour of light during its travel through the biological tissue. This enables the estimation of the relative oxygen saturation and blood perfusion in different layers of the tissue to be calculated, which has the potential to be a useful diagnostic tool.
\end{abstract}

Keywords: imaging photoplethysmography, pulse oximetry, oxygen saturation mapping, signal acquisition and processing.

*S.Hu@lboro.ac.uk; phone +44 1509227059

\section{INTRODUCTION}

Imaging photoplethysmography (iPPG) is one of the emerging medical imaging technologies to visualize peripheral blood perfusion in a specific tissue. Different from other optical related technologies, i.e., laser Doppler [1, 2] and Speckle imaging [3], iPPG aims to detect the dynamic change of blood volume in a designated tissue area rather than blood velocity and flow. Besides, iPPG can provide other human vital bioinformatics such as heart rate variability (HRV) [4] and pulse transit time (PTT) [5]. iPPG has significant priority over to the conventional PPG, as removes the primary limitations of spot measurement and contact sensory. iPPG can also monitor these vital human signs on different parts of skin surface simultaneously and bring the possible new insights that might come from hemodynamic mapping even 3D oxygen saturation in the tissue segments.

Non-contact camera based iPPG has been well established with the visualization of blood perfusion [6], which demonstrated the simultaneous capture of PPG waveforms from the extremities at three wavelengths (660 nm, $840 \mathrm{~nm}$ and $905 \mathrm{~nm}$ ) in both transmission and reflection modes. Another work has reported a reflection mode capture of 'heart cycle-related' pulsatile variations using a CMOS camera [7], with its illumination at $660 \mathrm{~nm}, 810 \mathrm{~nm}$, and $940 \mathrm{~nm}$. An additional research study presented a camera-based system capable of capturing two PPG signals at two wavelengths simultaneously in a non-contact manner [8].

Oxygen saturation mapping in variety of tissue segments is one such concept which has seen some interesting and promising developments over the last few years [9,10,11]. The development of improved medical optical technologies and the improvements in the understanding of light propagation through tissue has enables the standard contact PPG to undergo an evolutionary change into an imaging-based technique. The basic concepts from iPPG are utilized using a medical camera-based platform to acquire physiological information without the requirement of a full-contact sensor. This has the potential to enable monitoring under conditions which are currently extremely challenging, such as directly observing the behavior of a healing wound or the oxygen saturation of a burned region. Additionally, the use of an 
imaging platform can enable monitoring of additional physiological parameters, such as HRV and PTT. The technique has the potential to be utilized as a real-time monitoring method, suitable for not only clinical environment but also home-based care through the reduced requirements with regards to cabling and positioning. However these vital human biometrical signs extracted from iPPG needs a powerful technical approach to meet the requirements of flexible and compatible signal/image processing.

This paper presents 1) opto-physiological modelling-based iPPG engineering approach and 2) a biometric signal and image processing platform (bBIPP) developed at Loughborough University to enable not only layered blood perfusion but also tissue oxygen saturation for an individual in variety of tissue segments. The study has tightly connected with the iPPG setup together hardware with software components, each of which was designed to maximize the physiological signals acquired from the biological tissue objectives. The hardware element is designed to provide a stable and powerful illumination source for a high-speed CMOS camera which has the capability to record at a sufficient speed and resolution to enable the extraction of useful information. The software platform makes use of the recorded data, performing filtering operations to provide a clean signal for analysis and then extracting a relative saturation and perfusion for a region of interest.

This study is directed towards a single key aim, to show experimentally the feasibility for the use of a reflectance-mode imaging platform to acquire oxygen saturation $\left(\mathrm{SaO}_{2} \%\right)$ information from the body, and to demonstrate the overall accuracy and reliability for the technique.

\section{METHODS}

\subsection{Opto-physiological modelling driven imaging photoplethysmography}

The Beer-Lambert law defines the absorbance A of tissue as the natural log of the ratio of the intensity of the incident light to the transmitted light:

$$
A=-\ln \left(\frac{I_{0}}{I}\right)=\mu_{a} \times l
$$

If scattering is present, the incident light is attenuated due to both scattering and absorption. Even with $\mu_{a}=0$, there is attenuation because of the scattering of light away from the detector. The opto-physiological model introduces a scattering related path length term into the Beer-Lambert law, and a second term Is which represents the intensity of the light arriving at the detector when $\mu_{a}=0$ :

$$
A=-\ln \left(\frac{I}{I_{s}}\right)=\mu_{a} \times M P L
$$

where $M P L$ is the mean path length with unit of $\mathrm{mm}$.

$M P L$, yielded by the Monte Carlo (MC) simulation, takes account of anisotropic scattering, as well as the characteristics of the light source and the detector, and the geometry of tissue:

$$
M P L=(S+G) \times l\left(\mu_{s}, g\right)
$$

where $S$ is a factor that accounts for the position and size of the light source and the detector; $G$ is a factor that accounts for a specific tissue model including the geometry and the layered-interaction; $1\left(\mu_{s}, g\right)$ represents the path length modulated by the wavelength-dependent scattering coefficient $\mu_{\mathrm{s}}\left(\mathrm{mm}^{-1}\right)$ and the anisotropy factor $g$.

MPL is derived from the MC simulation presented in Fig. 1. Model preparation was performed in MATLAB (Mathworks Co., USA) using "STL" function to export the 3-D CAD data of a constructed anatomical tissue model into OptiCAD. The main process of MC ray tracing was performed by OptiCAD (Opticad Co., USA), which outputs coordinates of the traced ray vertices from the tissue model onto a file. Post data processing was performed in MATLAB through the use of custom algorithms specific to the outputs of the previous phases. 


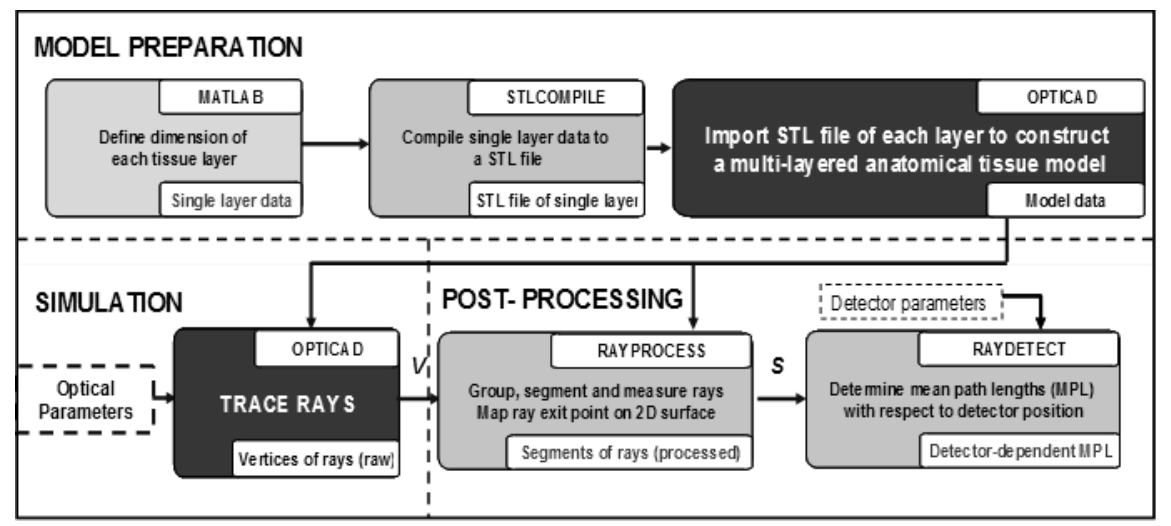

Fig. 1 The block diagram of MC simulation procedures where MATLAB functions are labeled in grey blocks and OPTICAD in black blocks.

To extend the opto-physiological model (OPM) into a multi-layered format, the tissue is treated as a composite medium $M=\{m(1), m(2) \ldots m(k)\}$ composed of $k$-layer media $(k=1,2 \ldots) . M P L(i)$ is the mean path length for each layer $m(i)$. Each layer is defined by a set of optical coefficients such as $\mu_{a, i}, \mu_{s, i}, g_{i}, n_{i}$ and the geometry such as the thickness $d i$ as shown in Fig. 2.

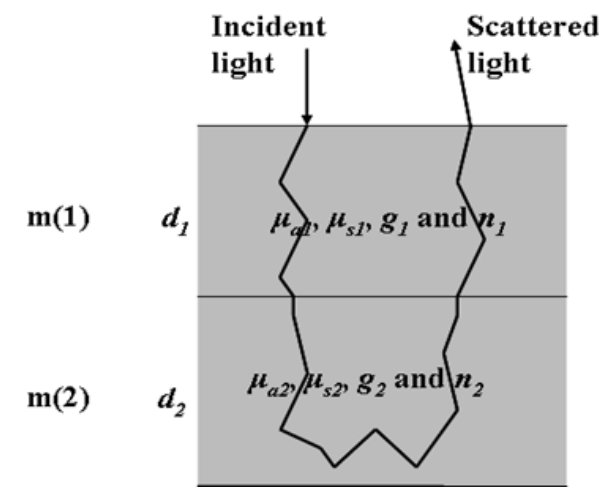

Fig. 2 A diagram of a two-layered tissue model $M=\{m(1), m(2)\}$.

The layered absorbance $A(i)$ can be derived from Eq. 2 according to the absorption coefficient $\mu_{a}(i)$ of the medium:

$$
A(i)=\mu_{a}(i) \times M P L(i)
$$

Following Eq. 4, the system absorbance of light propagation in the $n$-layered media can be derived as:

$$
A=\sum_{i=1}^{n} A(i)=\sum_{i=1}^{n}\left[\mu_{a}(i) \times M P L(i)\right]
$$

Based upon Eq. 5, the absorbance of multi-layered tissue can be quantified by the layered optical properties.

Blood perfusion is defined using the same formula as shown in Eq. 5, rewriting to show the effect of mean dynamic path length gives:

$$
I_{\text {perfusion }}(\lambda)=\sum_{i=1}^{k}\left(\mu_{a, \text { blood }}(i) \times M P L_{\text {dynamic }}(i)\right)
$$

Rearrangement of Eq. 6 allows the blood perfusion for a single tissue layer to be calculated in isolation: 


$$
I_{\text {perfusion }}(\lambda, i)=I_{\text {perfusion }}(\lambda) \times \frac{M P L_{\text {blood }}(i)}{\sum_{k} M P L_{\text {blood }}(k)}
$$

Blood oxygen saturation $\left(\mathrm{SaO}_{2} \%\right)$ is defined using the formula:

$$
S a O_{2}=\frac{R \mu_{H b}\left(\lambda_{2}\right)-\mu_{H b}\left(\lambda_{1}\right)}{R\left(\mu_{H b}\left(\lambda_{2}\right)-\mu_{H b O_{2}}\left(\lambda_{2}\right)\right)-\left(\mu_{H b}\left(\lambda_{1}\right)-\mu_{H b O_{2}}\left(\lambda_{1}\right)\right)}
$$

Where $R$, the ratio of ratios, is defined as:

$$
R\left(\lambda_{1}, \lambda_{2}\right)=\frac{I_{\text {perfusion }}\left(\lambda_{1}\right)}{I_{\text {perfusion }}\left(\lambda_{2}\right)}
$$

Similarly, by considering the process of iPPG from a technical standpoint, each individual pixel recorded from the camera can be given a coordinate and evaluated as if it were a single point source. By giving each pixel an $\mathrm{x}$ and $\mathrm{y}$ coordinate and amalgamating the results an image is created which provides a representation of the perfusion or saturation.

Extending Eq. 6 provides a representation for 2-dimensional blood perfusion within a region of interest.

$$
I_{\text {perfusion }}(\lambda, x, y)=\sum_{i=1}^{k}\left(\mu_{a, \text { blood }}(i) \times M P L_{\text {dynamic }}(i, x, y)\right)
$$

By taking the area-dependent perfusion map and combining it with the formula for ratio of ratios, a location-dependent value can additionally be calculated.

$$
R\left(x, y, \lambda_{1}, \lambda_{2}\right)=\frac{I_{\text {perfusion }}\left(x, y, \lambda_{1}\right)}{I_{\text {perfusion }}\left(x, y, \lambda_{2}\right)}
$$

Finally, a flat map for oxygen saturation can be obtained by making minor modifications to Eq.8 through the use of the modified form of $R$ obtained in Eq. 11.

$$
\mathrm{SaO}_{2}(x, y)=\frac{R\left(x, y, \lambda_{1}, \lambda_{2}\right) \mu_{H b}\left(\lambda_{2}\right)-\mu_{H b}\left(\lambda_{1}\right)}{R\left(x, y, \lambda_{1}, \lambda_{2}\right)\left(\mu_{H b}\left(\lambda_{2}\right)-\mu_{H b O_{2}}\left(\lambda_{2}\right)\right)-\left(\mu_{H b}\left(\lambda_{1}\right)-\mu_{H b O_{2}}\left(\lambda_{1}\right)\right)}
$$

Equation 7 defines perfusion for a single tissue layer, and can be used to further modify Eq.9 to provide knowledge for the behavior of multi-layered tissue.

$$
R\left(\lambda_{1}, \lambda_{2}, i\right)=\frac{I_{\text {perfusion }}\left(\lambda_{1}\right) \times w_{\text {perfusion }}\left(i, \lambda_{1}\right)}{I_{\text {perfusion }}\left(\lambda_{2}\right) \times w_{\text {perfusion }}\left(i, \lambda_{2}\right)}
$$

By merging Eq. 8 and Eq. 13 a formula for layered oxygen saturation can be obtained:

$$
\mathrm{SaO}_{2}(i)=\frac{R\left(\lambda_{1}, \lambda_{2}, i\right) \mu_{H b}\left(\lambda_{2}\right)-\mu_{H b}\left(\lambda_{1}\right)}{R\left(\lambda_{1}, \lambda_{2}, i\right)\left(\mu_{H b}\left(\lambda_{2}\right)-\mu_{H b O_{2}}\left(\lambda_{2}\right)\right)-\left(\mu_{H b}\left(\lambda_{1}\right)-\mu_{H b O_{2}}\left(\lambda_{1}\right)\right)}
$$


This formula can be further extended to provide detailed information for the perfusion and saturation at specific locations when using an imaging approach to data acquisition. By making the assumption that the perfusion and saturation within a region is constant, and defining an array of regions which can be individually analyzed, it becomes possible to map the locations of both sets of physiological data.

$$
I_{\text {perfusion }}(x, y, \lambda, i)=I_{\text {perfusion }}(x, y, \lambda) \times w_{\text {perfusion }}(i, \lambda)
$$

Taking the mapped perfusion data from Eq.12 allows the calculation of mapped ratio-of-ratios (Eq. 13), and by extension oxygen saturation (Eq. 14).

$$
\begin{gathered}
(x, y, i)=\frac{I_{\text {perfusion }}\left(x, y, \lambda_{1}\right) \times w_{\text {perfusion }}\left(x, y, i, \lambda_{1}\right)}{I_{\text {perfusion }}\left(x, y, \lambda_{2}\right) \times w_{\text {perfusion }}\left(x, y, i, \lambda_{1}\right)} \\
\mathrm{SaO}_{2}(x, y, i)=\frac{R(x, y, i) \mu_{H b}\left(\lambda_{2}\right)-\mu_{H b}\left(\lambda_{1}\right)}{R(x, y, i)\left(\mu_{H b}\left(\lambda_{2}\right)-\mu_{H b O_{2}}\left(\lambda_{2}\right)\right)-\left(\mu_{H b}\left(\lambda_{1}\right)-\mu_{H b O_{2}}\left(\lambda_{1}\right)\right)}
\end{gathered}
$$

By combining the individual regions of interest, it is possible to map the oxygen saturation of the whole image by recombining the individual points into a composite, showing the effects of each separate layer as a component overall saturation. By reducing the size of the regions of interest the resolution of the saturation map will be increased and show more detail, at the cost of much greater computational time.

\subsection{Biometric Signal and Image Processing Platform (bSIPP)}

The Biometric Signal and Image Processing Platform (bSIPP) is an extensible software environment built in MATLAB and designed for spatiotemporal $(S-T)$ processing of biometric image sequences. The bSIPP is an on-going development that aims for a simple way to store, deploy, communicate, modify and expand the signal and image processing work of one or more programmers. It is motivated by the need for an easier way to relay work from one engineer - or group of engineers - to another, and to work across disciplines.

The bSIPP comprises four data processing stages, namely the detection of raw data and delineation of datasets (RAW); the division, storage and preparation of datasets for pre-processing (PRE); the extraction and storage of S-T information from datasets (PROC); and the generation of custom data for display via manipulation of the S-T information (POST). The RAW stage involves detecting compatible image sequences within a root directory and its subdirectories on a storage device; determining the dimensions and parameters of the raw data, and determining the extents of all dimensions for PRE, PROC and POST data storage.

The PRE stage involves reading the raw frames of a dataset into memory with optional motion compensation; subdividing the raw image sequence into time-wise data segments (quadrants) and optionally storing these as quadrant files; generating 'compressed' quadrants by applying a 2x2 pixel averaging window with no overlapping to the data in the base quadrant and subsequent averaged quadrants up to the iteration that produces quadrants of length and width equal to 1 .

The PROC stage involves producing maps and arrays of maps from signal data contained in PRE storage (quadrants). Each signal is sequentially read and passed through various processing pipelines to produce scalars or vectors corresponding to each pixel position, yielding maps (scalar pipelines) or arrays of maps (vector pipelines). For pipelines involving correlation, i.e., correlation-coefficient $(\mathrm{cc})$ and cross-correlation (time shift, ts), each signal (or portion thereof) is passed with the corresponding reference signal.

The POST stage involves operations on the storage units (data fields) produced in PROC. Maps are produced and stored in memory on request. 


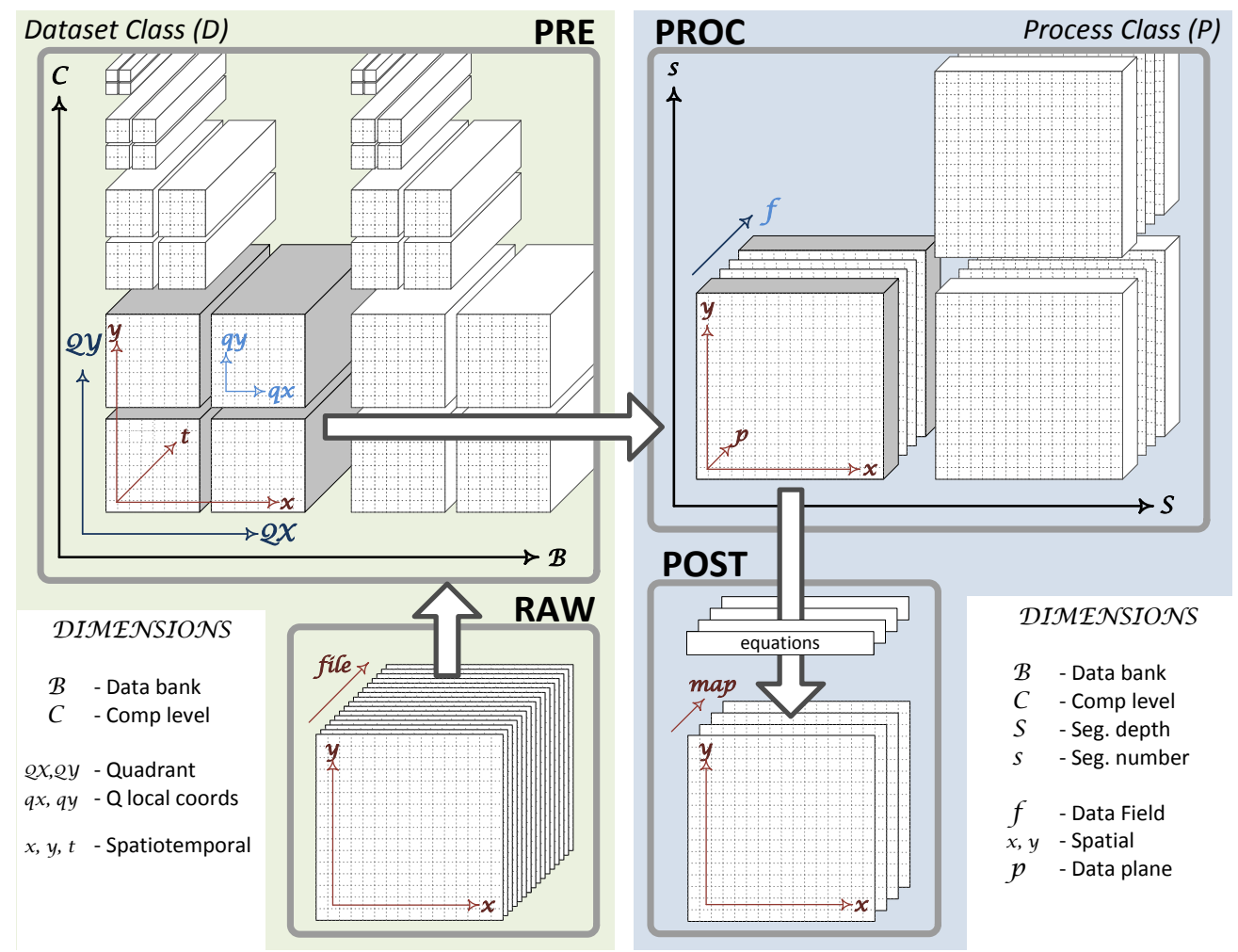

Fig. 3 Schematic overview of bSIPP dataset handling architecture.

\subsection{Instrumentation and protocol}

To enable the acquisition of multi-layered perfusion and saturation data there were some minor but significant changes made to both the hardware used in the experiment and to the methodology used. The most notable changes in the platform are the change from a CCD-style camera to a CMOS camera (MC1311, Mikrotron GmbH) and an increase in the number of RCLEDs in the ringlight, from 10 at $650 \mathrm{~nm}$ to 100 at $650 \mathrm{~nm}$ (TRC650SMD0603, WelTek Co. Ltd., Taiwan) and 100 at $870 \mathrm{~nm}$ (TRC870SMD0603, WelTek Co. Ltd., Taiwan). The parabolic form factor remains, as it was shown to provide a high level of uniformity to the area under illumination. To enable the illumination sources to be independently driven whilst maintaining a constant level of light a custom built constant-current switching power supply was added into the platform, utilizing an Arduino-based microcontroller to control and synchronize the light channels with the camera capture signal. The remainder of the platform is unchanged, with the same lens being used and a minor variation in the LabVIEW program during capture. 


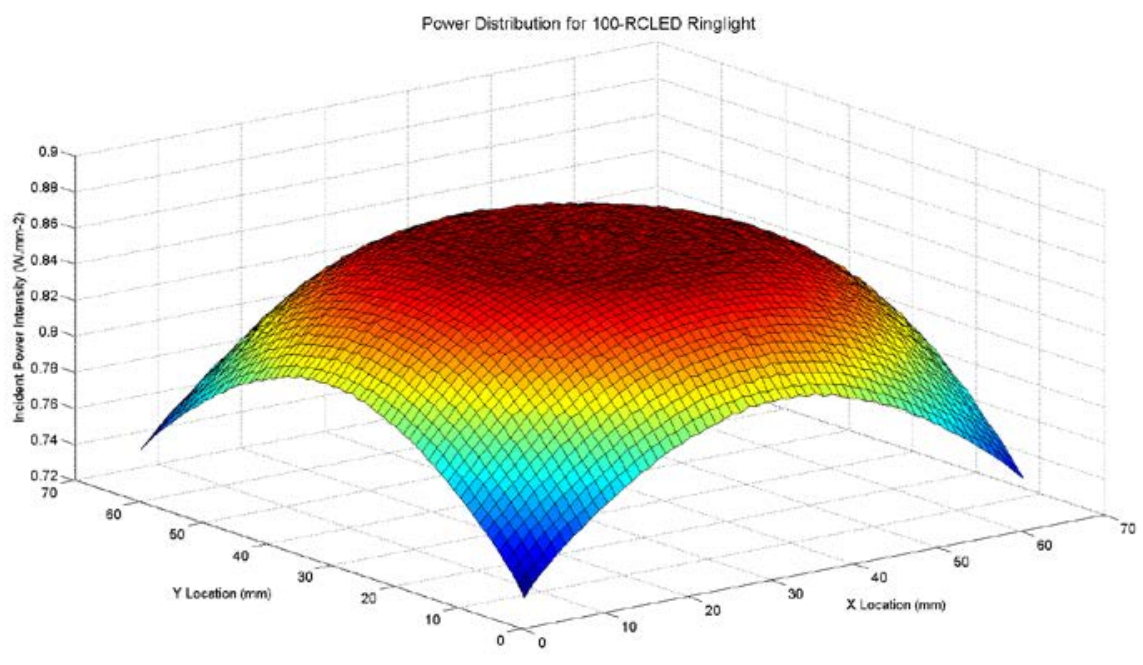

Fig.4 shows the modelled illumination intensity for the improved 100 RCLED ringlight for a $70 \mathrm{~mm} \times 70$ $\mathrm{mm}$ region, which surrounds the focal point of the camera. This clearly shows a high level of uniformity in incident power, which helps ensure any changes in acquired signal are from the subject.

The experimental process was also changed somewhat for the capture of physiological perfusion information, to account for variances in individual biology. Prior to the implementation of the measurements, the approval of the experimental protocol was obtained from the Loughborough University Ethical Committee. The procedures of the protocol can be briefly described as the following:

1) The subject under examination was asked to position his/her hand and forearm on an opaque, matte black support platform approximately $70 \mathrm{~mm}$ from the camera lens (the variation being a product of physical sizing). The subject was then asked to relax and remain motionless for five minutes to allow the circulatory system to stabilize.

2) The ringlight was enabled to provide a illumination at both $650 \mathrm{~nm}$ and $870 \mathrm{~nm}$, for alternating frames, and 30 seconds of 2-channel interleaved data were captured using the CMOS camera.

3) The subject under test was asked to perform 10 minutes of gentle exercise on a static exercise bike (approximately $20 \mathrm{kmh}^{-1}$ ) before a second set of data was captured.

4) The subject was allowed to rest for a further 10 minutes, and a third set of data was recorded.

5) By obtaining multiple datasets from individual subjects, changes in circulatory response and overall oxygen saturation could be acquired between datasets, further highlighting the versatility of the imaging approach.

The results for multiple tissue layers are a product of the iPPG data being used in conjunction with the theoretical formula shown in Eq. 17 and weighting values obtained from medical research to predict how the blood perfusion and oxygen saturation vary at different physical locations. The calculations are performed by bSIPP entirely within MATLAB to convert the flat imaged recorded into a multi-layered representation.

\section{RESULTS}

\subsection{D Perfusion mapping}

The behaviour of the blood flow varies with the location of tissue in the human body, by utilizing the experimental techniques outlined it becomes possible to map the blood flow and show how it varies from location to location. 


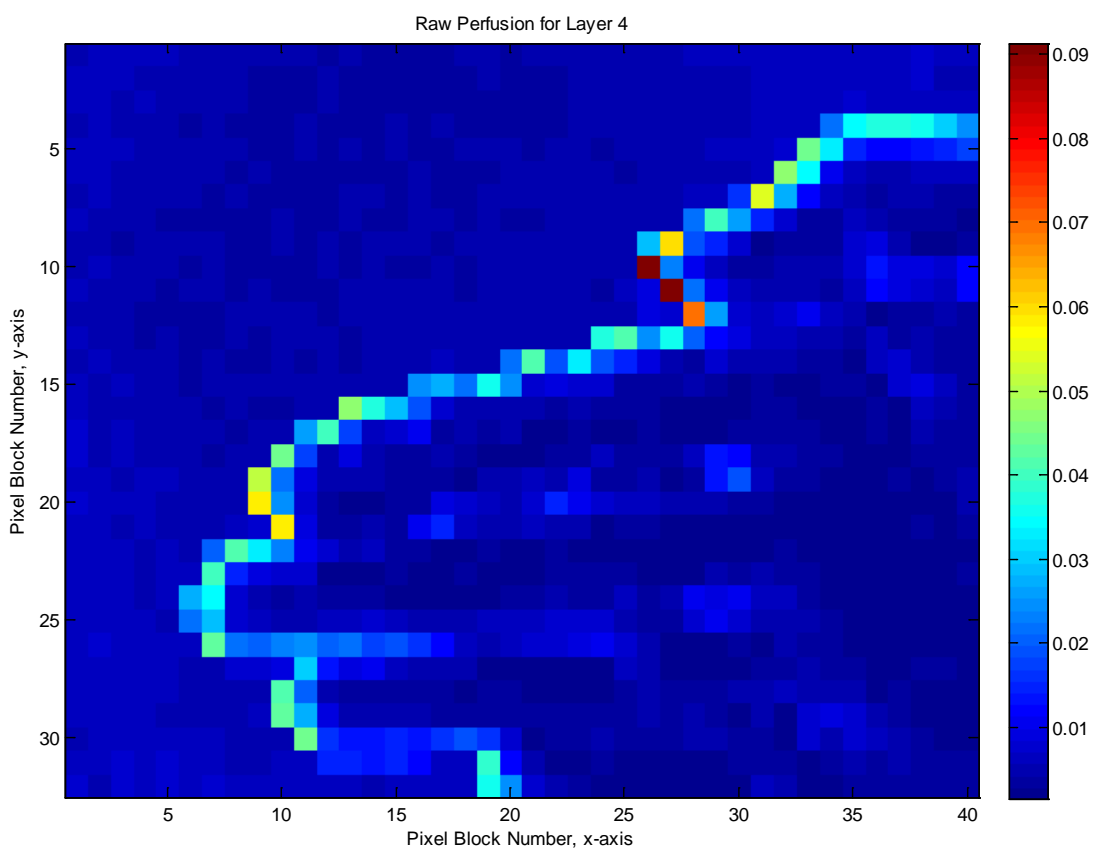

Fig. 5 Results from a single dataset for layered perfusion. Although the outline of the hand distorts the results somewhat, variation is clearly visible between the differing parts of the human hand. Further modification to the analysis suite should allow this to be overcome and more clear data to be extracted.

\subsection{Multi-layered oxygen saturation $\left(\mathrm{SaO}_{2} \%\right)$}

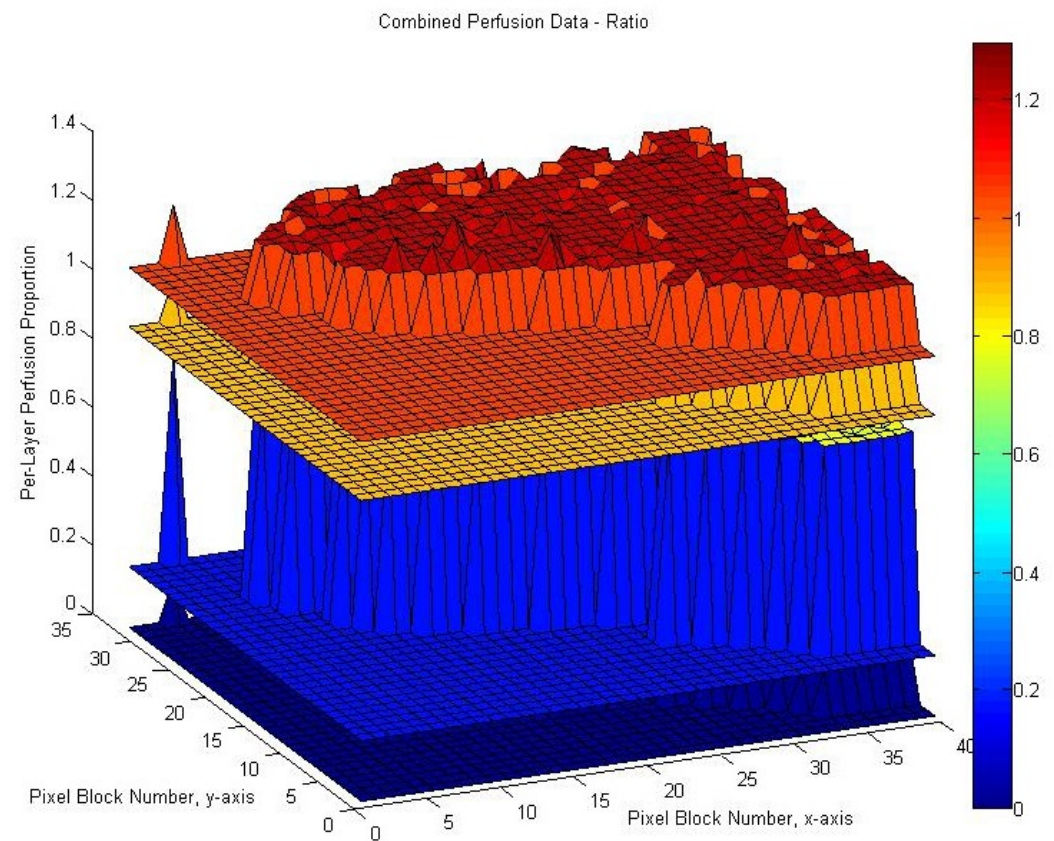

Fig. 6 Stacked results for layered blood perfusion. The results clearly show a large variation in the proportional volume of blood for different tissue layers, from lowest to highest: Subdermis, Dermis Plexus Profundus, Deep Dermis, and Dermis Plexus Superficialis. 


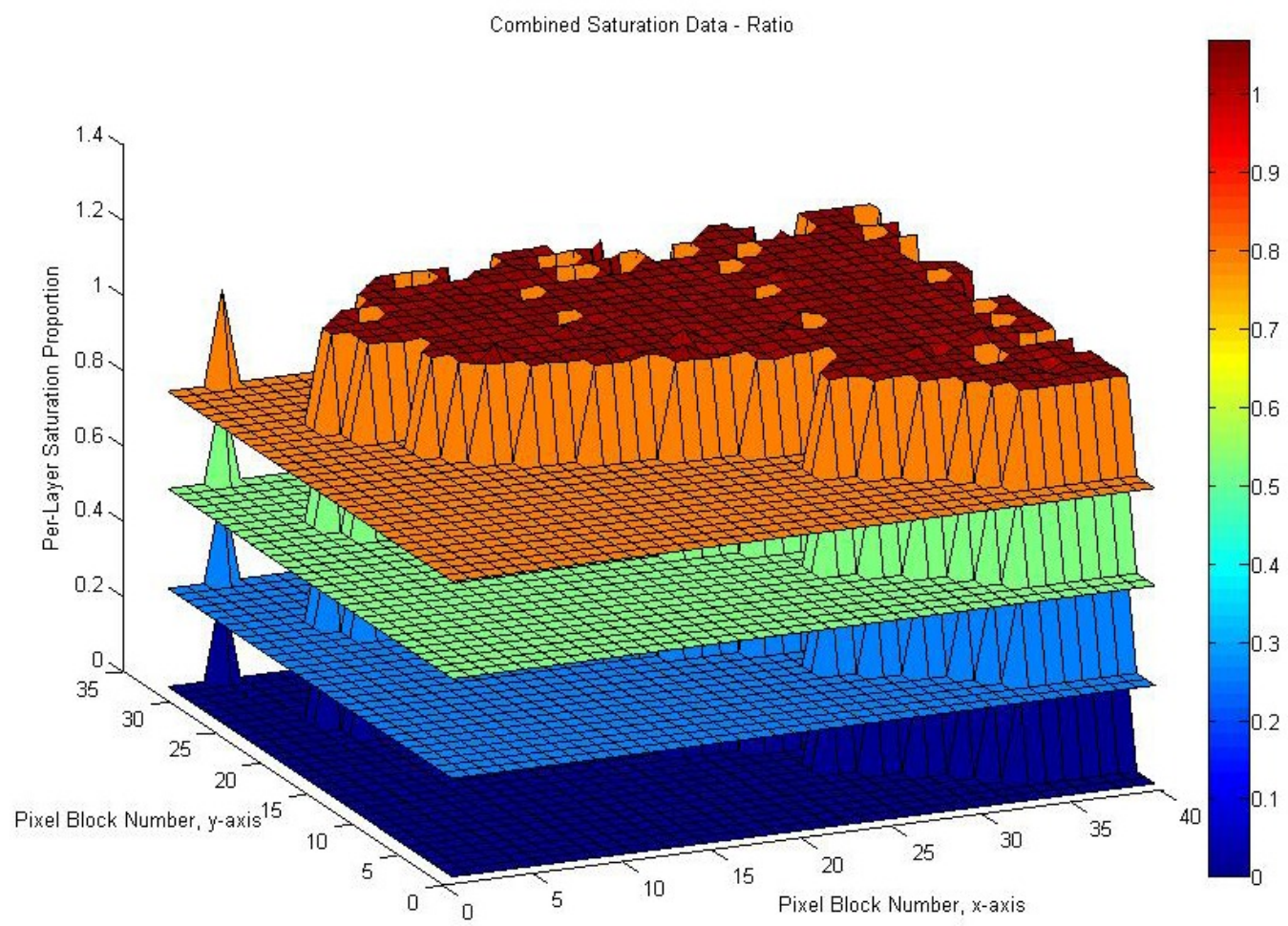

Fig. 7 Layered representation of blood saturation. The image clearly shows how the distribution of oxygen by tissue layer varies from the proportions of blood perfusion. Despite having notably different volumes of blood, the saturation remains almost constant throughout the different tissue layers. This image shows the relative proportion of the signal when compared to the total at each grid point.

When directly comparing the results for the multiple layered saturation and perfusion, the expected behaviour obtained from theory matches the results shown; in that the blood oxygen saturation levels remain constant throughout the various tissue layers as shown by the near identical proportion whereas the blood perfusion is strongly coupled with the physical layer structure. By extending the existing theories into the practical domain it can be shown that the iPPG technique can extract an increased amount of information when compared to other contact approaches. Further development of the modelling techniques and improved experimental configuration should enable increased volumes of data to be acquired and with higher resolution between layers.

One important aspect when considering iPPG techniques is the overall reliability and accuracy when compared to the standard contact techniques. When the iPPG technique is to become widely used and medically viable it must perform to the same level of accuracy as existing techniques, and as a result the experimental configuration used has provided a direct means to perform an initial comparison. 

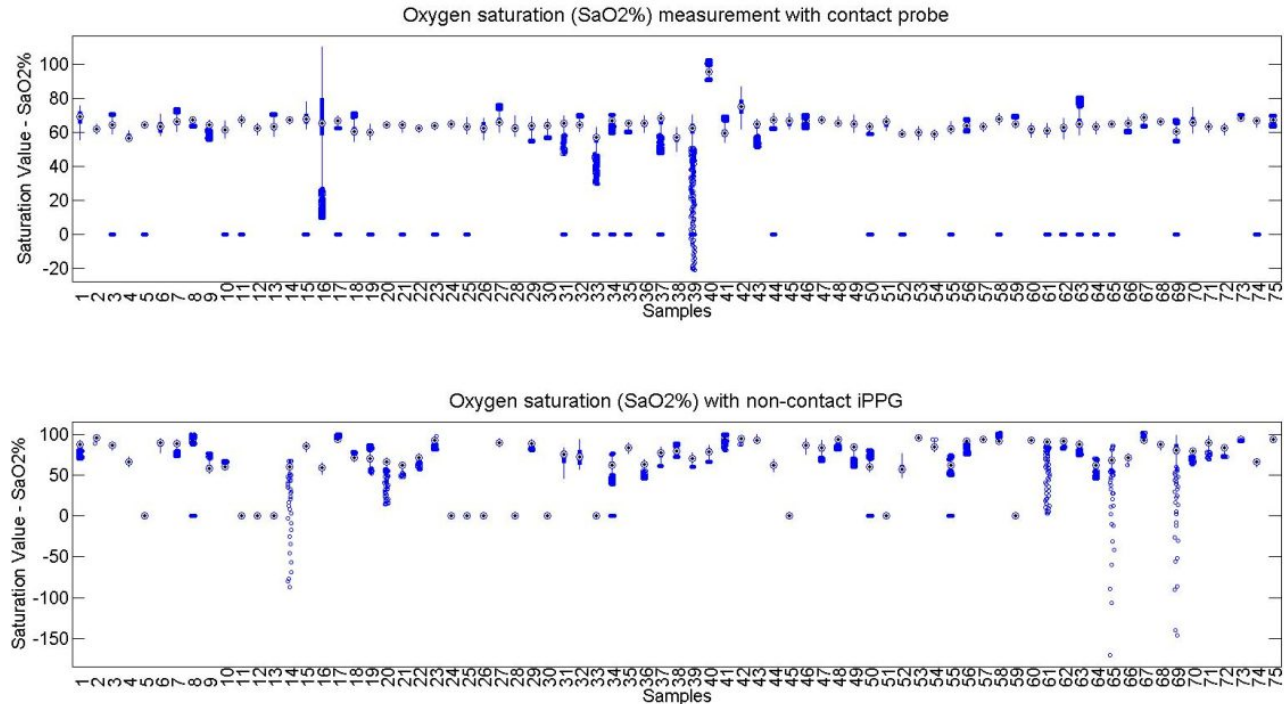

Fig 8: shows the mean total oxygen saturation over 30 seconds for the standard contact PPG system and the iPPG platform. Although the standard deviation of the imaging platform is almost double that of the contact platform ( 0.0247 for the contact, and 0.0445 for the imaging), a reasonable correlation for the saturation between platforms can be observed (0.8247). One major point of note is that the contact platform shows a far lower saturation than expected, which is likely through a lack of calibration against a known phantom. Additionally, some results are highly skewed on the imaging platform, which is likely caused through motion-artefact effects, which is an area for future work.

\section{DISCUSSION AND CONCLUSIONS}

The OPM is created by redefining the path length of the Beer-Lambert law. The redefined path length, referred to as mean path length (MPL), is derived through the outputs of the OPM on multi-layered tissue with layered optical properties and specified geometries. The simplified representation of the OPM eliminates the mathematic complexity of some current propagation models such as radiative transport equation [8] and thus it can be applied in complicated tissue. On the other hand, the accuracy of the model prediction increases by taking scattering into account. The realization of the modeling is similar to the modified Beer-Lambert law [12] which has been widely used to quantify the concentration of tissue chromospheres by a ratiometric approach. Differently, the OPM quantifies the blood perfusion in a multilayered format by treating the tissue in a multi-layered structure with respective blood volume changes towards multilayered $\mathrm{SaO}_{2} \%$, rather than a whole and static tissue.

The use of an iPPG platform for the extraction of multi-layered blood perfusion and $\mathrm{SaO}_{2} \%$, is shown to be conceptually viable, based upon preliminary experimental results by means of OPM. Whilst the iPPG currently does not match the gold standard of contact platforms regarding accuracy or precision, the potential to extract detailed information from a patient using a relatively simple technique means that further development should be undertaken. Significant areas of note where gains can be made are in the software domain, where optimization and advancements in image processing may allow higher resolution images to be handled more easily, and with compensation for some of the more common motion artefacts. In addition, improved theoretical models of physical tissue structure and optical interactions within them will provide a more solid basis for defining the parameters used in the calculation of layered blood perfusion and $\mathrm{SaO}_{2} \%$.

\section{ACKNOWLEDGEMENT}

The authors are grateful to all the members in the Photonics Engineering and Health Technology Research Group of Loughborough University for their enthusiastic support. Also, the authors would like to thank the financial support of Loughborough University and the RCLEDs supplier WelTek Co. Ltd., Taiwan. 


\section{REFERENCES}

[1] Wardell K, Nilsson G E, “Duplex Laser Doppler Perfusion Imaging,” Microvasc. Res., 52, 171-182 (1996).

[2] Chen Z P, Milner T E., Srinivas S, Wang X J, Malekafzali A, VanGemert M J C, Nelson J S, "Noninvasive imaging of in vivo blood flow velocity using optical Doppler tomography," Optics Letters, 22(14), 1119-1121 (1977).

[3] Zakharov P, Völker A C, Wyss M T, Haiss F, Calcinaghi N, Zunzunegui C, Buck A, Scheffold F, Weber B., "Dynamic laser speckle imaging of cerebral blood flow," Opt. Express, 17(16),13904-13917 (2009).

[4] Lu G, Yang F, Taylor J A Stein J F, "A comparison of photoplethysmography and ECG recording to analyse heart rate variability in healthy subjects,” J. Med. Eng. Technol., 33(8), 634-641(2009).

[5] Loukogeorgakis S, Dawson R, Phillips N, Martyn C N, Greenwald S E, "Validation of a device to measure arterial pulse wave velocity by a photoplethysmographic method,” Physiol. Meas., 23, 581-596 (2002).

[6] Zheng J, Hu S, "The preliminary investigation of imaging photoplethysmographic system," J. Physics: Conference Series, 85, 012031(2007).

[7] Wieringga F P, Mastik F, Van Der Steen A FW, "Contactless multiple wavelength photoplethysmographic imaging: a first step towards ‘SpO2 camera’ technology,” Ann. Biomed. Eng., 33(8), 1034-1041 (2005).

[8] Humphreys K, Ward T, "Noncontact simultaneous dual wavelength photoplethysmography: A further step toward noncontact pulse oximetry," Rev. Sci. Instrum., 78, 044304(2007).

[9] Li J, Dunmirea B, Beachb K W, Leotta D F, "A reflectance model for non-contact mapping of venous oxygen saturation using a CCD camera”, Optics Comm., 308,78-84(2013).

[10] Shafique M, Kyriacou P A, "Photoplethysmographic signals and blood oxygen saturation values during artificial hypothermia in healthy volunteers,” Physiol. Meas., 33(12), 2065-78 (2012).

[11] Lázaro J, Gil E, Bailón R, Mincholé A, Laguna P, "Deriving respiration from photoplethysmographic pulse width,” Med. Biol. Eng. Comput., 51(1-2), 233-42 (2013).

[12] Hu S, Azorin-Peris V, Zheng J, "Opto-physiological modeling applied to photoplethysmographic cardiovascular assessment,” J. Healthc. Eng., 4(4), 505-28 (2009). 